\title{
The Extracellular Chaperone Clusterin Potently Inhibits Human Lysozyme Amyloid Formation by Interacting with Prefibrillar Species
}

\author{
Janet R. Kumita ${ }^{1}$, Stephen Poon ${ }^{1}$, Gemma L. Caddy ${ }^{1}$ \\ Christine L. Hagan ${ }^{1}$, Mireille Dumoulin ${ }^{1}$, Justin J. Yerbury ${ }^{2}$ \\ Elise M. Stewart ${ }^{2}$, Carol V. Robinson ${ }^{1}$, Mark R. Wilson ${ }^{2}$ \\ and Christopher M. Dobson ${ }^{1 *}$
}

${ }^{1}$ Department of Chemistry University of Cambridge Cambridge CB2 1EW, UK

${ }^{2}$ School of Biological Sciences University of Wollongong Wollongong 2522, Australia

\begin{abstract}
We have studied the effects of the extracellular molecular chaperone, clusterin, on the in vitro aggregation of mutational variants of human lysozyme, including one associated with familial amyloid disease. The aggregation of the amyloidogenic variant I56T is inhibited significantly at clusterin to lysozyme ratios as low as 1:80 (i.e. one clusterin molecule per 80 lysozyme molecules). Experiments indicate that under the conditions where inhibition of aggregation occurs, clusterin does not bind detectably to the native or fibrillar states of lysozyme, or to the monomeric transient intermediate known to be a key species in the aggregation reaction. Rather, it seems to interact with oligomeric species that are present at low concentrations during the lag (nucleation) phase of the aggregation reaction. This behavior suggests that clusterin, and perhaps other extracellular chaperones, could have a key role in curtailing the potentially pathogenic effects of the misfolding and aggregation of proteins that, like lysozyme, are secreted into the extracellular environment.
\end{abstract}

(C) 2007 Elsevier Ltd. All rights reserved.

Keywords: human lysozyme; systemic amyloidosis; clusterin; chaperones; fibril formation

\section{Introduction}

Protein misfolding that results in the formation of aggregates is associated with a range of human diseases, including both organ-specific conditions, such as Alzheimer's disease and type II diabetes, and systemic conditions, such as the non-neuropathic amyloidoses associated with the proteins transthyretin and lysozyme. ${ }^{1}$ Since 1993, six naturally occurring lysozyme variants associated with amyloid disease (I56T, F57I, F57I/T70N, W64R, D67H and T70N/W112R), ${ }^{2-5}$ and one non-amyloidogenic variant $(\mathrm{T} 70 \mathrm{~N}){ }^{6}$ have been discovered. In-depth characterization of two of the disease-associated

Present address: S. Poon, School of Biological Sciences, University of Wollongong, Wollongong, 2522, Australia.

Abbreviations used: TEM, transmission electron microscopy; Thio-T, thioflavin-T.

E-mail address of the corresponding author: cmd44@cam.ac.uk variants, I56T and D67H, as well as the non-diseaserelated $\mathrm{T} 70 \mathrm{~N}$ variant, has been performed using a variety of biophysical techniques. ${ }^{7-14}$ Deposits of $\mathrm{I} 56 \mathrm{~T}$ and $\mathrm{D} 67 \mathrm{H}$ formed in vitro possess the key characteristics of amyloid fibrils; these include their fibrillar morphology in TEM images, their displays of birefringence upon binding the dye Congo red and a typical cross- $\beta$ X-ray diffraction pattern. ${ }^{10-12}$ The kinetics of in vitro fibril formation by both I56T and $\mathrm{D} 67 \mathrm{H}$ are sigmoidal, showing a lag phase followed by an exponential growth phase typical of amyloidogenic systems. ${ }^{10-12}$ Furthermore, fibril formation by I56T, D67H and wild-type lysozymes can be accelerated by seeding with pre-formed fibrils, ${ }^{12}$ indicating that lysozyme fibril formation proceeds through a nucleation-dependent mechanism. ${ }^{15}$

The native state of lysozyme consists of two structural regions, one predominantly helical (the $\alpha$-domain) and the other containing significant $\beta$-sheet structure (the $\beta$-domain). The amyloidogenic nature of the disease-associated lysozyme variants has been found to result from a reduction 
in native state stability and cooperativity, which allows the population of a transient and aggregation-prone partially unfolded intermediate under physiologically relevant conditions. In this intermediate, the $\beta$-domain and an adjacent helix (C-helix) of the $\alpha$-domain are unfolded simultaneously, whereas the regions forming the remainder of the protein fold maintain their native-like structure., ${ }^{9,11}$

The ability of proteins to misfold, and even to form organized aggregated species such as amyloid fibrils, is inherent in their nature and can occur in vivo either during the initial folding process following biosynthesis, or later as a result of the intrinsic fluctuations in their conformations. ${ }^{16}$ The systemic amyloidoses are of particular interest, as pathogenicity appears to be determined primarily as a consequence of the disruption of vital organs by large, often massive, accumulations of aggregated proteins. ${ }^{17}$ Biological systems have therefore evolved a variety of mechanisms to avoid such aggregation under normal conditions. As well as the evolutionary design of the sequence of amino acids, these protective mechanisms include quality control and degradation processes, and the presence of a wide range of molecular chaperones. ${ }^{18}$ The latter are of considerable importance; however, the vast majority of known chaperones are found inside the cell, whilst the bulk of proteinaceous aggregates accumulate in extracellular space. ${ }^{19,20}$ It is therefore of particular importance, in the context of amyloid disease, that three extracellular chaperones have been recently discovered. ${ }^{19}$

The best characterized of these molecular chaperones is clusterin, a $\sim 61 \mathrm{kDa}$ glycoprotein that is translated as a 449 amino acid residue single polypeptide chain, the first 22 of which represent a secretory signal that is proteolytically cleaved after biosynthesis. ${ }^{21,22}$ Post-translational modification results in an $\mathrm{N}$-linked glycosylated protein that is also cleaved proteolytically into an $\alpha$ and $\beta$-peptide that are concomitantly linked by five disulfide bonds to form a heterodimer. ${ }^{22}$ Clusterin is distributed widely throughout the body, with average concentrations in human plasma of $100( \pm 50) \mu \mathrm{g} / \mathrm{ml}$, although levels in human seminal fluid are about ten times higher. ${ }^{23,24}$ The precise physiological role of clusterin is unknown, although a large diversity of functions has been suggested. ${ }^{25}$ In particular, it has been shown that the chaperone behavior of clusterin can be observed in vitro and appears similar to that described for the small heat shock proteins. ${ }^{26,27}$ Clusterin has been reported to inhibit in vitro fibril formation by a number of proteins and peptides linked to aggregation diseases including the $A \beta$ peptide, apo C-II, and the prion neuropeptide 106126. ${ }^{19,40}$

A wide range of biophysical and biochemical techniques has been used over some years to explore, in vitro, the molecular mechanism by which disease-associated variants of human lysozyme form amyloid fibrils, and to relate these findings to the likely origin of the familial systemic amyloidosis with which extracellular deposits of these species are associated. ${ }^{9,10}$ In the present study we have utilized our extensive knowledge of this system to examine the effects of clusterin on the aggregation reaction of a globular protein, and hence to explore the possible significance in the prevention of widespread deposition of extracellular proteins in living systems.

\section{Results}

The process of fibril formation in the present study has been explored by investigating the amyloidogenic lysozyme variant, I56T, as well as a nonnatural variant of human lysozyme, I59T, which possesses the same mutation in a similar region of the structure, but which has greater stability and cooperativity than the natural amyloidogenic variants (our unpublished results). For both proteins, amyloid fibrils were observed to form at $\mathrm{pH} 5.0$ and they appeared similar to those reported previously (Figures 1 and 2). In order for fibrils to form on suitable time-scales for these experiments, temperatures of $48{ }^{\circ} \mathrm{C}$ (for I56T) and $60^{\circ} \mathrm{C}$ (for I59T) were chosen. Using a light-scattering assay, we initially monitored the aggregation of each protein in the presence and in the absence of substoichiometric amounts of clusterin over time-spans of $90 \mathrm{~h}$ for I56T (Figure 1(a)) and $10 \mathrm{~h}$ for I59T (Figure 2(a)). In the absence of clusterin, fibrils formed readily in both cases, with half-times for the aggregation process of $26( \pm 6) \mathrm{h}$ for I56T and $2.8( \pm 0.6) \mathrm{h}$ for I59T. In the presence of a 1:40 clusterin-to-lysozyme molar ratio, the changes in light-scattering were essentially completely absent for both variants on these timescales. Complete inhibition over the time-courses studied here was also found with clusterin-tolysozyme molar ratios of 1:4, 1:8 and 1:80. The observed inhibitory effect was specific to the presence of clusterin and was not seen upon addition of similar ratios of conalbumin or bovine serum albumin (BSA) to lysozyme undergoing aggregation (data not shown). At the ends of the incubation times, each sample was analyzed by transmission electron microscopy (Figures 1(b) and 2(b)) and the fluorescence of the amyloid-binding dye, thioflavin T was measured (Figures 1(c) and 2(c)). In addition, the samples were passed through $0.22 \mu \mathrm{m}$ pore size filters followed by analysis of the flow-through by SDS-PAGE to determine the quantities of soluble protein present in the solutions (Figures 1(d) and 2(d)). These procedures revealed that the samples of I56T and I59T in the absence of clusterin had converted almost completely into amyloid fibrils. However, in each of the samples containing clusterin, no such fibrillar aggregates were observed and a large proportion of the lysozyme molecules (>95\%) were still present in solution. Some non-fibrillar aggregates could be observed in the lysozyme samples containing clusterin, but similar species were visible also in clusterin samples in the absence of the lysozyme 

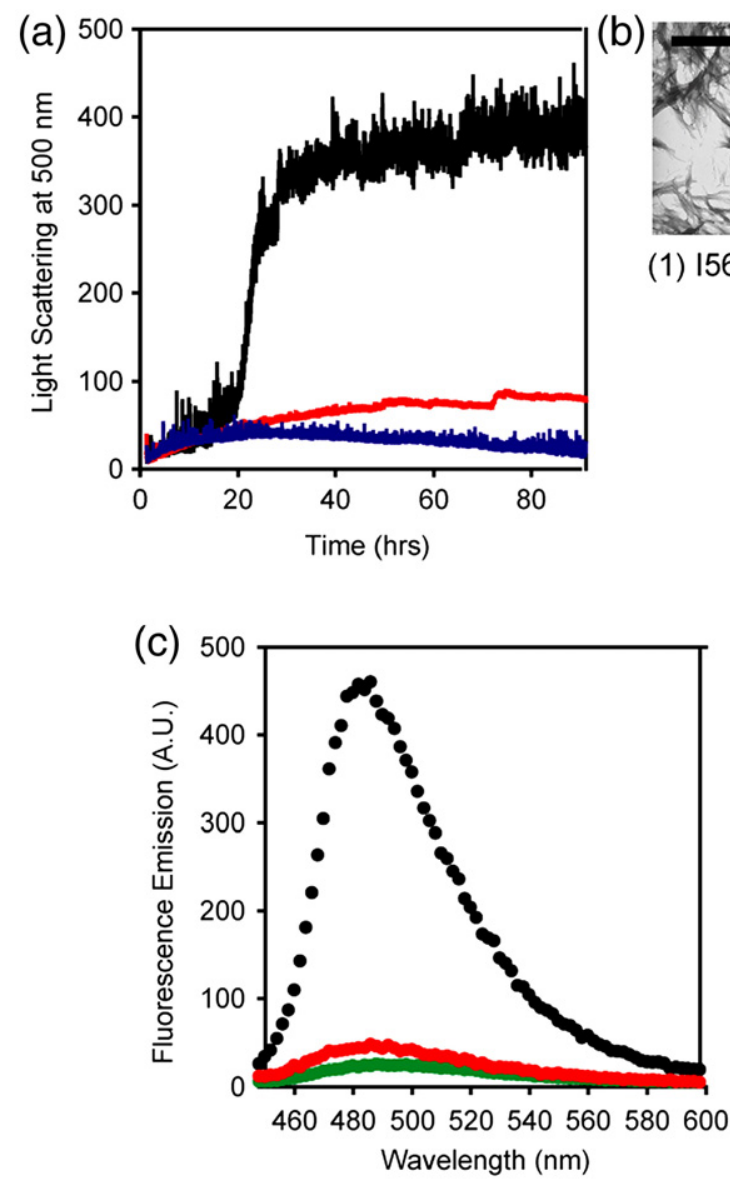

(b)

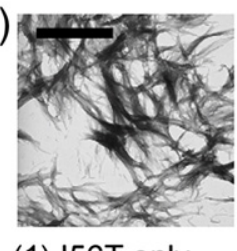

(1) I56T only

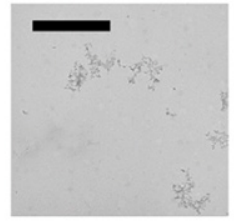

(2) I56T:clusterin (40:1)

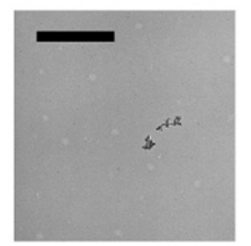

(3) clusterin alone

(d)

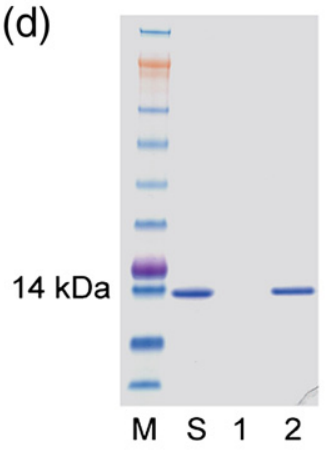

Figure 1. Inhibition of I56T lysozyme fibril formation by clusterin. (a) Light-scattering results from I56T lysozyme aggregation in the absence (black) and presence of clusterin (red) at a 1:40 clusterin-to-lysozyme molar ratio. The lightscattering intensity for clusterin alone is shown in blue. Samples were incubated with stirring at $48^{\circ} \mathrm{C}$ and light-scattering was monitored at $500 \mathrm{~nm}$. (b) TEM analysis of samples taken at the end of the growth phase observed in the lightscattering assay. The scale bar represents $1 \mu \mathrm{m}$. (c) Thioflavin-T fluorescence measured at the end of the growth phase observed in the light-scattering assay for buffer only (green), I56T lysozyme alone (black) and I56T lysozyme with clusterin (red). (d) SDS-PAGE analysis of samples at the end of the growth phase observed in the light-scattering assay after being passed through $0.22 \mu \mathrm{m}$ pore size filters. No soluble protein remained in the I56T sample in the absence of clusterin (lane 1); in contrast, the majority of lysozyme remained in the soluble form in the sample containing clusterin (lane 2). Molecular mass markers are shown in lane $\mathrm{M}$, and an equal volume of a soluble I56T lysozyme solution $(7 \mu \mathrm{M})$ is shown in lane S.

variants, albeit in smaller quantities. These species may, therefore, be the result of slow aggregation of clusterin as well as of lysozyme itself under our conditions.

In order to probe the mechanism of inhibition of aggregation, we first examined the interaction of clusterin with the native state of lysozyme by means of size-exclusion chromatography and ELISA assays. No species of increased mass (representing a lysozyme/clusterin interaction) was detectable by either assay; even in solutions containing equimolar ratios of clusterin-to-lysozyme; i.e. at 80 times the level at which clusterin was observed to inhibit fibril formation (Figure 3). We then probed the interaction of clusterin with the transient, partially unfolded lysozyme species that has been identified as a precursor to amyloid aggregation. ${ }^{28}$ To achieve this objective, we monitored the rates of solvent exchange of labile hydrogen in the protein by mass spectrometry, under conditions where the exchange rates are fast compared to the local co-operative unfolding process that generates the aggregationprone intermediate (i.e. EX1 conditions); the presence and nature of the latter can then be monitored directly. ${ }^{9}$ Moreover, as well as characterizing the intermediate, we have used this approach to show that the inhibition of fibril formation by an antibodybinding domain results from the stabilization of the cooperative nature of the folded native state, which in turn inhibits the formation of the transient intermediate. ${ }^{10}$ We therefore compared the hydrogen exchange behavior of I56T lysozyme in the absence and in the presence of clusterin (Figure 4(a)). The results reveal that even at high clusterin-tolysozyme ratios (1:1.5), the rate of formation of the intermediate and the number of protected amides is not detectably altered by the presence of clusterin (Figure 4(b)). Thus, even under these conditions, I56T lysozyme is still able to populate the transient intermediate, whose properties appear unchanged, 
(a)

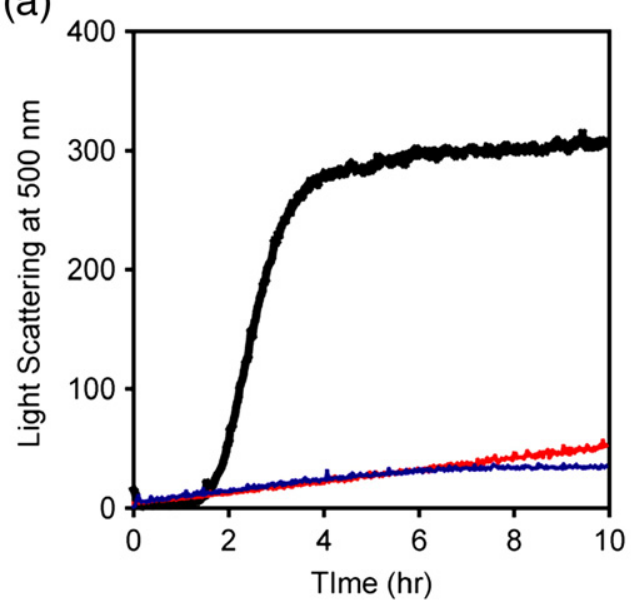

(c)

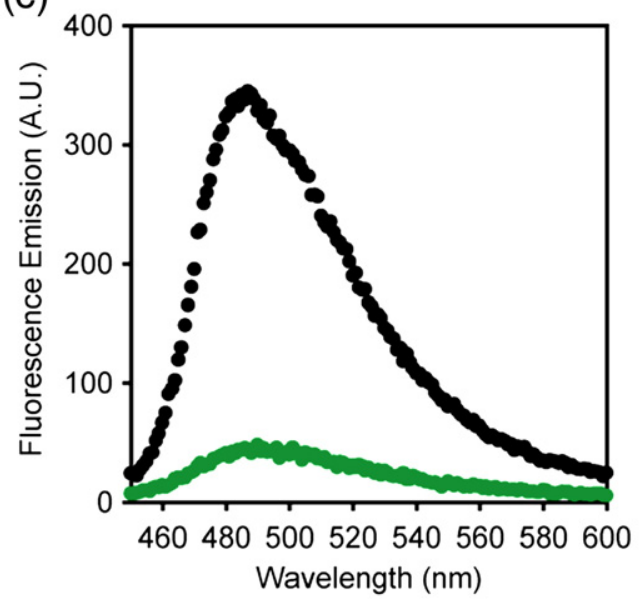

(b)

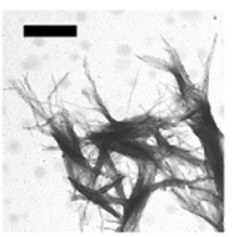

(1) 159T only

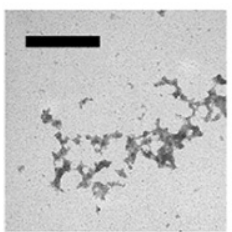

(2) 159T:clusterin (40:1)

(d)

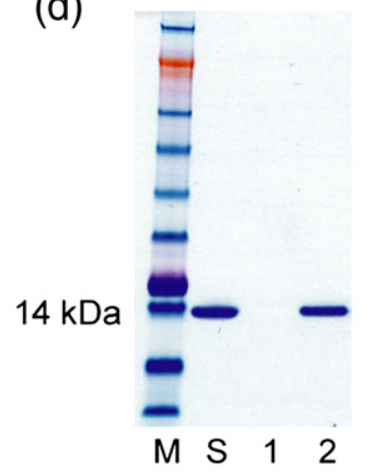

Figure 2. Inhibition of I59T lysozyme fibril formation by clusterin. (a) Light-scattering results from I59T aggregation in the absence of clusterin (black) and with a molar ratio of 1:40 clusterin-tolysozyme (red). The light-scattering intensity for clusterin alone is shown in blue. Samples were incubated with stirring at $60^{\circ} \mathrm{C}$ and the light-scattering was monitored at $500 \mathrm{~nm}$. (b) TEM analysis of samples taken at the end of the growth phase observed in the light-scattering assay. Clusterin alone under these conditions showed species identical with those seen in Figure 1(b). The scale bar represents $500 \mathrm{~nm}$. (c) Thioflavin-T fluorescence measured at the end of the growth phase observed in the light-scattering assay for buffer only (green) and I59T alone (black). (d) SDS-PAGE analysis of samples at the end of the growth phase observed in the light-scattering assay after being passed through $0.22 \mu \mathrm{m}$ pore size filters. No soluble protein remained in the I59T sample in the absence of clusterin (lane 1); however, the majority of lysozyme remained in the soluble form in the sample containing clusterin (lane 2). Molecular mass markers are shown in lane $\mathrm{M}$, and an equal volume of a soluble I59T lysozyme solution $(7 \mu \mathrm{M})$ is shown in lane $\mathrm{S}$. indicating that the inhibitory effect of clusterin on fibril formation does not result from its influence on this intermediate state but on a step that is subsequent to its formation in the aggregation pathway.

As it has been shown that clusterin does not interact detectably with monomeric lysozyme or the monomeric transient intermediate, but is able to inhibit fibril formation when added at submolar ratios at the beginning of the reaction, we attempted to define the stage of the aggregation reaction at which clusterin is most effective. Addition of substoichiometric amounts of clusterin at the beginning of the reaction results in the inhibition of fibril formation over the entire timecourse of the reaction that we analyzed. When, however, the same amount of clusterin was added mid-way through the elongation phase of lysozyme fibril formation, the degree to which clusterin inhibits aggregation was much less than that resulting from the earlier addition (Figure 5). It can be concluded, therefore, that the species recognized by clusterin must be populated in the lag phase of fibril formation and that, once fibril elongation and growth has begun, the effect of clusterin is substantially compromised.
The inhibitory effect of clusterin must therefore occur subsequent to the formation of the transient intermediate but before the elongation phase. In order to narrow down the species involved still further, we studied the non-native variant of human lysozyme, I59T, which, under the fibril-forming conditions used for this variant, possesses a highly reproducible lag phase of about $2.8 \mathrm{~h}$, to probe for the presence of clusterin/lysozyme complexes. We attempted this task first by using a dot blot analysis, in which samples of different time-points during the lag phase of lysozyme were incubated with clusterin. However, no complex was detected reproducibly at the sensitivity we were able to achieve in this assay. In order to gain additional sensitivity as well as to search for the complex in situ, we instead incubated a 1:8 molar ratio of clusterin-to-lysozyme under fibril-forming conditions, and removed aliquots at different time-points for analysis in microtiter plate wells containing an immobilized monoclonal anti-clusterin antibody. After incubation, the wells were washed and probed with a lysozyme-specific antibody; a positive result indicated the presence of a complex. Using this approach, we were able to detect a complex demonstrating that clusterin does indeed interact with one or more types of species populated during the fibril 
(a)
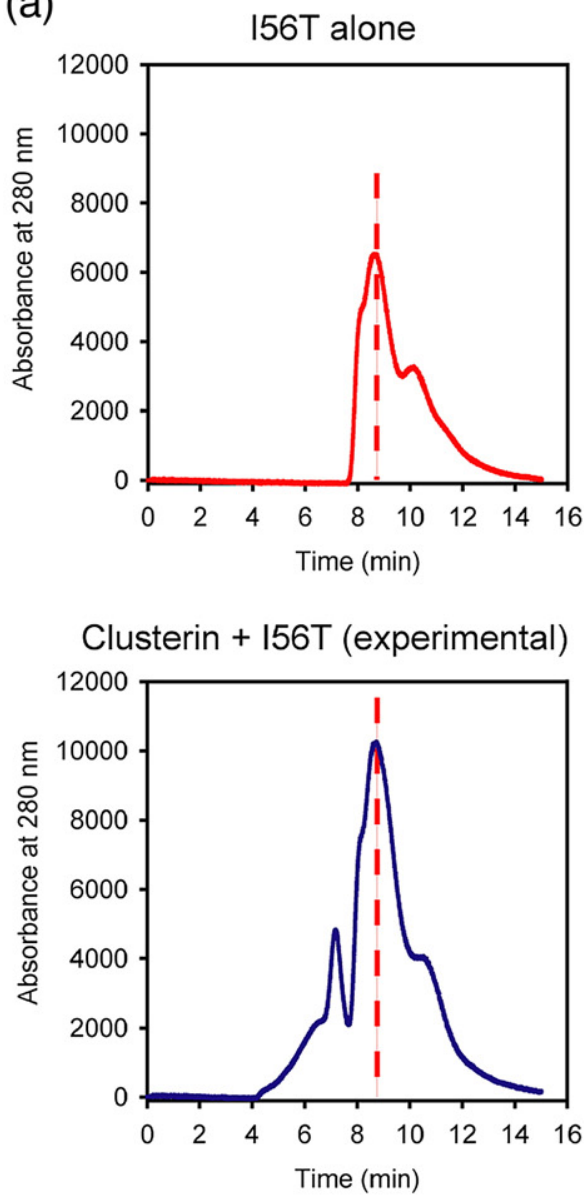
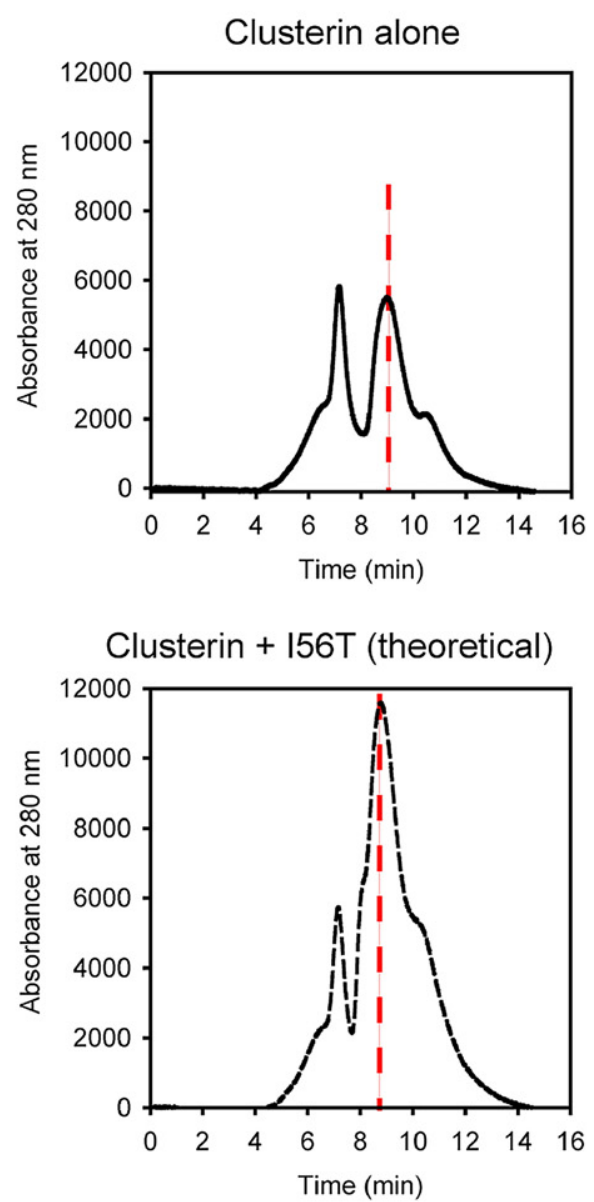

(b)

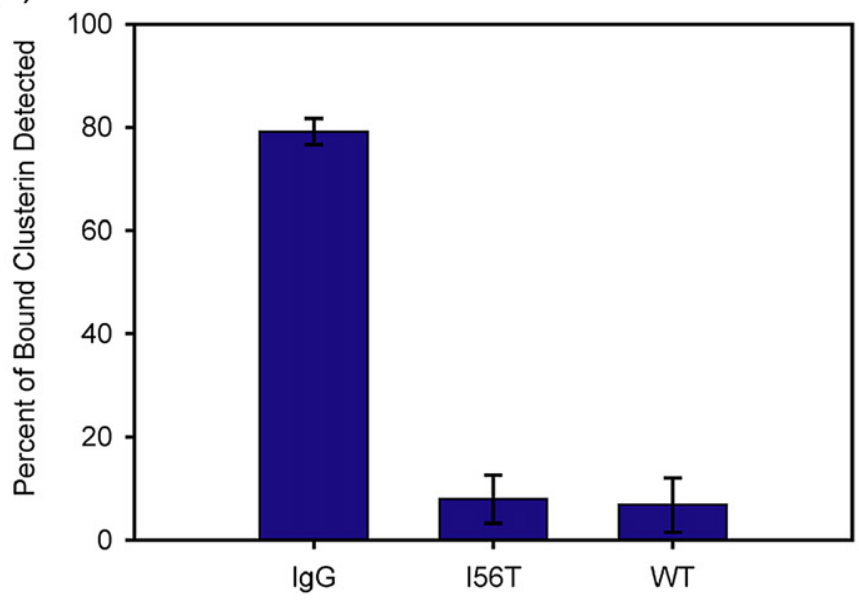

Figure 3. Analysis of interactions between clusterin and native I56T lysozyme. (a) Analysis by size-exclusion chromatography of clusterin alone, I56T alone, and a mixture of I56T and clusterin (at a 1:1 molar ratio). The result obtained for the mixture of clusterin and I56T (experimental) does not differ significantly from the theoretical result obtained by summing the traces for lysozyme alone and clusterin alone (i.e. assuming that there is no interaction between clusterin and I56T lysozyme). The dotted red line represents the centre of the buffer peak in all spectra. (b) Interactions between lysozyme and clusterin determined by ELISA. The graph displays the levels of clusterin bound to immobilized IgG (positive control) and I56T and wild-type lysozymes. All experiments were performed in triplicate.

lag phase (Figure 6). Analysis of the time-course of clusterin binding indicates that a complex with lysozyme is detected relatively late in the aggregation process. Indeed, binding appears at a time- point that, in the absence of clusterin, would be at the end of the elongation phase (4.8 h) (Figure 6). In the presence of clusterin, no significant lightscattering is observed at this time, or at time-points 
(a) I56T alone

\section{I56T with clusterin}

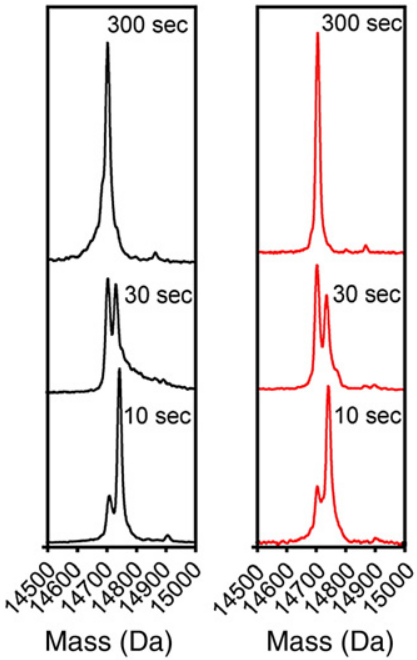

(b)

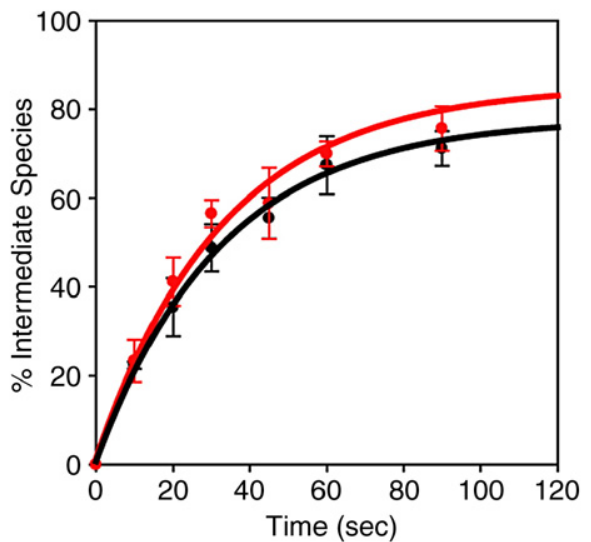

Figure 4. Effect of clusterin on the transient intermediate of lysozyme. (a) Time-points of hydrogen exchange reactions as monitored by mass spectrometry. In the presence and in the absence of a 1:1.5 molar ratio of clusterin-to-lysozyme, a higher mass species dominates initially (after $10 \mathrm{~s}$ of exchange) $(14,743.2$ $( \pm 1.1) \mathrm{Da})$. As the time of exchange increased, the intensity of the lower mass species $(14,704.2( \pm 3.7) \mathrm{Da})$ increases and by $300 \mathrm{~s}$ is the sole observable peak. The mass of deuterated I56T lysozyme is $14943.6 \mathrm{Da}$. (b) The population of intermediate is plotted versus time to determine the rate of formation of the transient intermediate. The half-times $\left(\tau_{1 / 2}\right)$ for I56T lysozyme in the absence (black) and presence (red) of clusterin are $18.2( \pm 2.4) \mathrm{sec}$ and 21.2 $( \pm 2.2) \mathrm{s}$, respectively.

that are twice as long. To ensure this signal is due to the presence of a complex and not due to binding of lysozyme aggregates to the microtiter plate wells, aliquots taken at different time-points from the samples containing lysozyme alone were investigated. It is clear from these experiments that there is no detectable non-specific binding of lysozyme aggregates in this assay (Figure 6(c)). It should be noted that the complex represents a very small fraction of the overall reaction mixture; when the flow-through was examined by SDS-PAGE, the amount of soluble lysozyme still present in the reaction mixture was greater than 95\% (Figure 6(b)). These findings therefore confirm that clusterin

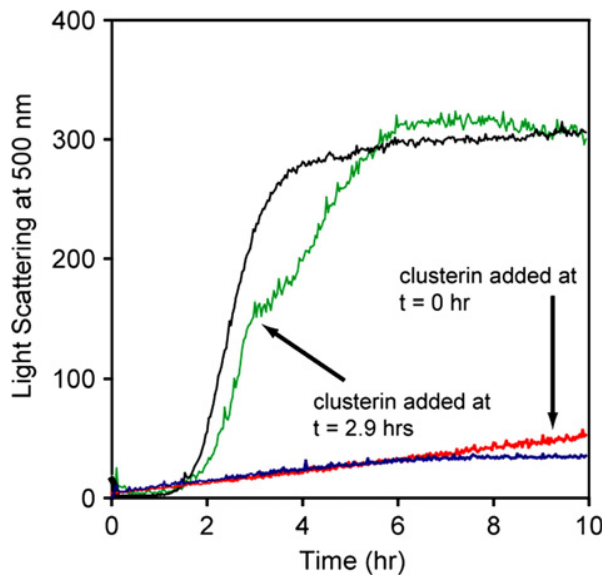

Figure 5. Effectiveness of the addition of clusterin at different time-points during the light-scattering assay. Light-scattering results of I59T lysozyme aggregation in the absence of clusterin (black), and following addition of clusterin to the I59T reaction in sub-stoichiometric quantities (1:40) at time zero (red) and at $\mathrm{t}=2.9 \mathrm{~h}$ (green). The time-dependence of the light-scattering for clusterin alone is shown in blue. recognizes and binds to a species along the lysozyme fibril-forming pathway, which with continued incubation under fibril-forming conditions, forms detectable complexes. Moreover, the fact that interactions with a very small fraction of the total soluble lysozyme present suppresses the aggregation process suggests that this species is a key intermediate on the route to fibril formation, and that in the early stages of inhibition, clusterin has the ability to reduce the population of this species formed from reaching the threshold that leads to fibril formation.

\section{Discussion}

Clusterin has a remarkable ability to inhibit lysozyme fibril formation even when present at a highly sub-stoichiometric molar ratio. The very low levels of clusterin that are sufficient to generate complete inhibition for prolonged periods of time, together with the lack of interaction between clusterin and native lysozyme, suggests strongly that clusterin exerts its effects by interacting with a species that is not highly populated, yet crucial for the aggregation process. Mass spectrometric analysis reveals that this species is not the transient and partially unfolded monomeric intermediate that has been identified as the initial step in the pathway of lysozyme amyloidogenesis. ${ }^{10,11}$ The kinetics of this latter process show a clear lag phase, followed by rapid exponential growth, typical of amyloid formation in other systems. ${ }^{15}$

Under the conditions used in the present study, complexes of clusterin with lysozyme species formed in situ early in the lag phase were not detectable, although in studies using much higher concentrations of lysozyme, and indeed other proteins, we have established that clusterin can 


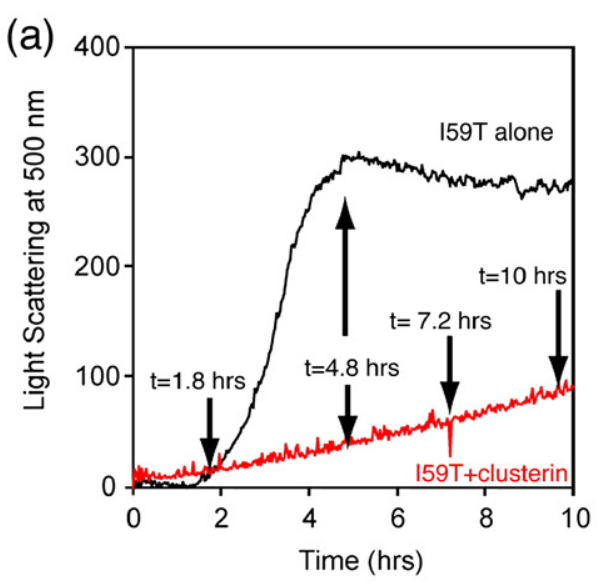

(b)

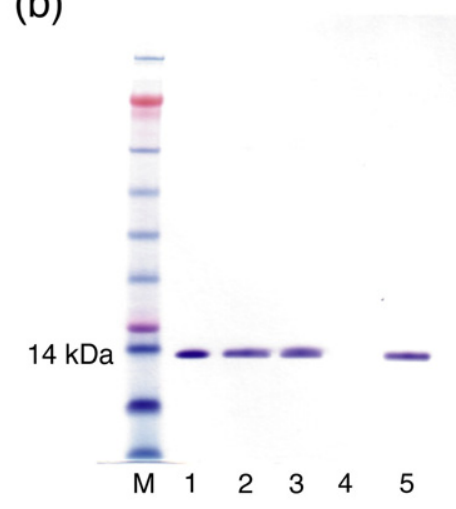

(c)

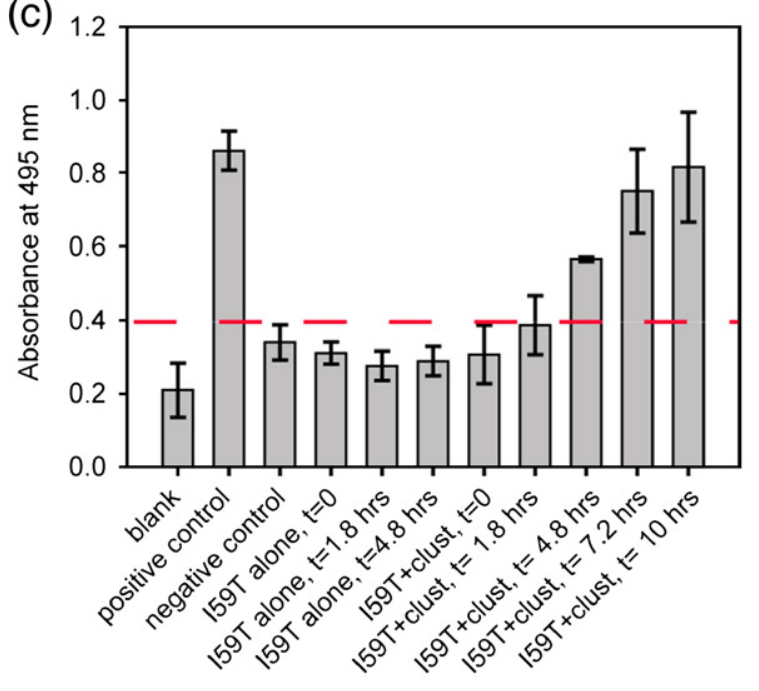

Figure 6. Detection of lysozyme/clusterin complex under fibril-forming conditions. $(\alpha)$ Lightscattering of $159 \mathrm{~T}$ in the absence of clusterin (black) and I59T in the presence of a clusterin-to-lysozyme molar ratio of 1:8 (red). The timepoints analyzed for the presence of complex are indicated. (b) SDSPAGE analysis of samples passed through a $0.22 \mu \mathrm{m}$ pore size filter. Samples include molecular mass markers (lane $\mathrm{M})$, soluble lysozyme (7 $\mu \mathrm{M})$ (lane 1), lysozyme/ clusterin samples at $4.8 \mathrm{~h}$ (lane 2), $7.2 \mathrm{~h}$ (lane 3), $10 \mathrm{~h}$ (endpoint) of I59T lysozyme alone (lane 4), $10 \mathrm{~h}$ (endpoint) of I59T with clusterin (lane 5). (c) Detection of the clusterin/lysozyme complex monitored at $495 \mathrm{~nm}$ after probing with anti-lysozyme antibodies. The blank contained no sample, the positive control had lysozyme adsorbed to the wells, and the negative control incubated lysozyme in wells adsorbed to the anti-clusterin antibody. The red dotted line represents the detected absorbance due to nonspecific binding based on the negative control. bind to preformed oligomeric species. The present results reveal, however, that the dominant species binding to clusterin accumulates to detectable levels later in the aggregation process. These species are, even at this stage of the reaction, present at very low levels, enabling highly sub-stoichiometric amounts of clusterin to inhibit aggregation effectively completely for lengths of time over which the lysozyme variant in the absence of clusterin, converts into amyloid fibrils. The lack of a stable complex early in the aggregation process is likely to be due, in part, to the lack of sensitivity in the detection methods. It is interesting, however, that we do not detect a complex until relatively late in the inhibition reaction, which may suggest that the interaction between clusterin and lysozyme in the early stages of aggregation may be transient in nature. In this case, the complex detected when lysozyme is in the presence of clusterin could arise from the further association of such species.

The oligomeric species populated during the phase of protein aggregation before the formation of fibrils are of particular interest, both because of their evident role in nucleating fibril growth, and because the relatively small oligomers involved in misfolded aggregates with high surface-to-volume ratios appear to be highly toxic. ${ }^{20}$ These species, are, however, intrinsically heterogeneous and the aggre- gates observed in the present study may represent, at least in part, the continuous association of small oligomers. ${ }^{20}$ Indeed, it appears extremely likely that clusterin prevents lysozyme aggregation by interacting with the variety of oligomeric species developing along the fibril-forming pathway. It is interesting to note, however, that recent studies have implicated oligomers of the size range observed in the present work as the most effective species inducing fibril formation, probably by nucleation of $\beta$-sheet formation within the larger oligomeric species. ${ }^{29}$ From the present results and other studies, this evidence suggests that clusterin is likely to interact preferentially with small soluble oligomers. $^{30,31}$

The inhibitory effect of clusterin is fully consistent with a model for fibril formation by human lysozyme, in which protein molecules overwhelmingly populate the native state, but structural fluctuations in the amyloidogenic variants can readily lead to a high enough population of partially unfolded monomeric precursors to initiate the aggregation process (Figure 7). ${ }^{32}$ The recent demonstration that inhibition of fibril formation can occur as a result of the binding of antibody fragments, preventing the formation of this transient monomeric species, supports the importance of this species as a precursor to oligomer formation. ${ }^{10}$ It is 
1) decreased native stability and reduction in cooperativity results in population of transient intermediate

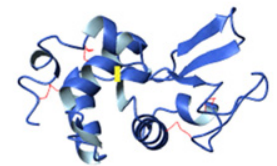

Native

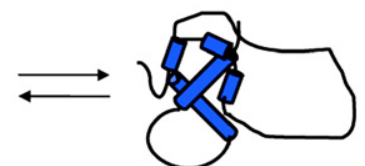

Intermediate

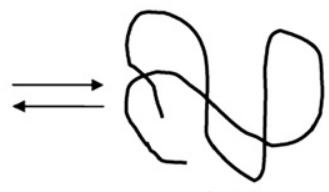

Unfolded

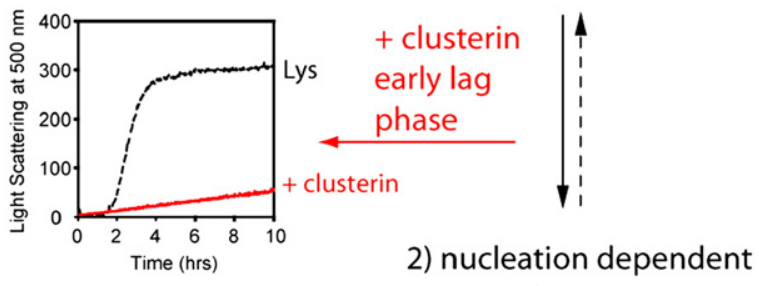

aggregation
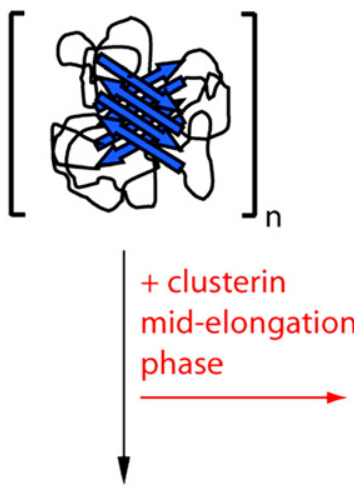

3) formation of amyloid fibrils
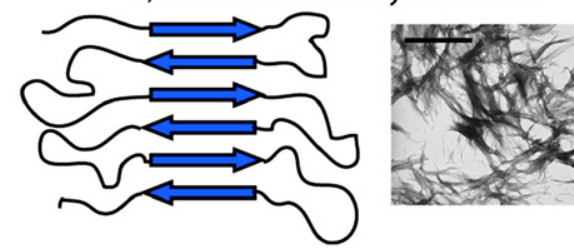

Figure 7. Proposed pathway for lysozyme fibril formation and it's inhibition by clusterin. Lysozyme exists primarily in its native state under physiological conditions. In the amyloidogenic variants, the decreased native state stability and reduction in cooperativity results in the transient formation of an intermediate at concentrations high enough to be detected using hydrogen exchange monitored by mass spectrometry. ${ }^{32}$ The formation of intermolecular interactions between such intermediates can generate oligomeric species that are subsequently able to nucleate fibril growth. The ways which clusterin could inhibit such events are indicated in red. therefore likely that the oligomeric species, which are likely to be highly heterogeneous, may be recognized in vivo by clusterin before the accumulation of extracellular amyloid deposits and the onset of amyloidosis.

The mechanism of inhibition of lysozyme amyloid formation by clusterin that has been deduced from the present study, along with the ubiquitous presence of relatively high levels of clusterin in different tissues, strongly supports the concept that clusterin has an important, and general, role in avoiding protein aggregation. Our results show that clusterin is most effective at inhibiting fibril formation when present early in the lag phase of the aggregation process, an observation consistent with the findings from studies of the inhibition of apolipoprotein C-II fibril formation by clusterin and by another molecular chaperone, $\alpha$-crystallin that is classified as a small heat-shock protein. ${ }^{30,31,33}$ Protein misfolding is not confined to a small number of proteins, but is an inherent consequence of the stochastic nature of the process of protein folding and refolding. ${ }^{1,16}$ Moreover, proteins have an innate tendency to aggregate under some conditions. It appears likely, therefore, that clusterin has an important role in suppressing such effects under normal physiological conditions.

The ability of clusterin to accumulate oligomeric species to form relatively stable complexes suggests that extracellular deposition of amyloid fibrils result, for example, in situations when larger than normal populations of misfolded proteins and oligomers appear. Such situations can be the result of mutations, such as in lysozyme amyloidosis, or other factors, such as an increase in protein levels e.g. of $\beta 2$-microglobulin in dialysis-related amyloidosis, ${ }^{34}$ or a decrease in the efficacy of the various protective mechanisms against aggregation that can occur in old age. ${ }^{35}$ Moreover, the key role played by clusterin in our mechanisms of defense against amyloid formation suggests that enhancement of its levels in tissue, or of its ability to sequester or induce clearance of aggregates, could be a potential therapeutic strategy. 


\section{Materials and Methods}

The I56T and I59T human lysozyme variants were expressed and purified as described. ${ }^{36,37}$ Human clusterin was purified from serum by immunoaffinity chromatography as described. ${ }^{38}$ For secondary purification, the eluate was dialyzed against $20 \mathrm{mM}$ Tris- $\mathrm{HCl}(\mathrm{pH} 8.0)$ and loaded onto a Q-Sepharose column. Bound proteins were eluted using a $0 \mathrm{M}-0.7 \mathrm{M} \mathrm{NaCl}$ gradient in the same buffer and clusterin-containing fractions were pooled and concentrated. For all proteins, stock solutions of lyophilized material were made in Milli-Q water. Stock solutions were passed through $0.22 \mu \mathrm{m}$ pore size filters and concentrations were determined by UV-visible spectroscopy as described; ${ }^{7}$ dilutions into appropriate buffers were made for each assay. Monoclonal anti-clusterin antibodies, G7 and 41D, were generated as described, ${ }^{39}$ and polyclonal anti-human lysozyme antibodies were prepared in rabbits by Washington Biotechnology Inc. (Simpsonville, MD, USA).

\section{Aggregation monitored by right-angle light-scattering}

Aggregation studies were performed with I56T human lysozyme $(6.8 \mu \mathrm{M}, 0.1 \mathrm{M}$ sodium citrate buffer $\mathrm{pH} 5.0)$, incubated with stirring at $48{ }^{\circ} \mathrm{C}$ in a Cary Eclipse spectrofluorimeter (Varian Ltd., Oxford UK), or with a non-natural lysozyme variant, I59T $(6.8 \mu \mathrm{M}, 0.1 \mathrm{M}$ sodium citrate buffer $\mathrm{pH} 5.0$ ) incubated at $60{ }^{\circ} \mathrm{C}$ with stirring. Light-scattering was monitored at $500 \mathrm{~nm}$ with slit-widths of $5 \mathrm{~nm}$. Inhibition of fibril formation by clusterin was monitored in the same fashion using a range of clusterinto-lysozyme molar ratios (1:80, 1:40, 1:8 or 1:4). These ratios were calculated from the monomeric molecular mass of the lysozyme variants $(14,680.6 \mathrm{Da}$ for both) and from a mass of $61 \mathrm{kDa}$ for clusterin based on the average of two reported glycoforms. ${ }^{21}$

\section{Thioflavin-T binding}

A stock solution of thioflavin-T (Thio-T) $(2.5 \mathrm{mM})$ was prepared in $10 \mathrm{mM}$ sodium phosphate buffer ( $\mathrm{pH}$ 7.0) containing $150 \mathrm{mM} \mathrm{NaCl}$. Fresh stock solutions were prepared daily and passed through $0.22 \mu \mathrm{m}$ pore size filters. Samples were prepared with $100 \mu \mathrm{l}$ endpoint aliquots in $0.1 \mathrm{M}$ sodium citrate buffer ( $\mathrm{pH}$ 5.0) containing $62.5 \mu \mathrm{M}$ Thio-T. Thio-T binding was measured in a Cary Eclipse spectrofluorimeter with an excitation wavelength of $440 \mathrm{~nm}$ (slit-width $5 \mathrm{~nm}$ ) and an emission scan from 450$600 \mathrm{~nm}$ (slit-width $5 \mathrm{~nm}$ ). All experiments were performed in triplicate unless stated otherwise.

\section{Transmission electron microscopy (TEM)}

Samples were applied to Formvar-coated nickel grids, stained with $2 \%(\mathrm{w} / \mathrm{v})$ uranyl acetate solution and viewed in a Philips CEM100 transmission electron microscope operating at $80 \mathrm{kV}$ as described. ${ }^{10}$

\section{SDS-PAGE analysis}

Samples were passed through a $0.22 \mu \mathrm{m}$ pore size PVDF filter. The filtrates were analyzed by SDS-PAGE using $4 \%-$ $12 \%$ Bis-Tris NuPAGE gels (Invitrogen UK) in Mes buffer under non-reducing conditions. Gels were stained using Coomassie brilliant blue.

\section{Hydrogen exchange monitored by electrospray ionization mass spectrometry}

I56T human lysozyme was deuterated at exchangeable sites by unfolding in deuterated guanidinium hydrochloride. $^{9}$ The proteins were refolded via dilution in ${ }^{2} \mathrm{H}_{2} \mathrm{O}$ and concentrated in a Vivaspin 20 concentrator (Sartorius Ltd., Epsom UK) with a molecular mass cutoff of $5 \mathrm{kDa}$. This cycle of dilution and concentration was repeated until no guanidinium ion contamination was observable by mass spectrometry. The final stock solutions of protein were $200 \mu \mathrm{M}$ in ${ }^{2} \mathrm{H}_{2} \mathrm{O}$. Deuterated I56T $\left(200 \mu \mathrm{M}\right.$ in $\left.{ }^{2} \mathrm{H}_{2} \mathrm{O}\right)$ was added to lyophilized clusterin to give a 1:1.5 clusterin-to-lysozyme molar ratio. Samples were prepared for hydrogen/deuterium (H/D) exchange by manual mixing as described. ${ }^{14}$ Spectra were acquired over a mass/charge range of 500 to 5000 Da on an LCT MS instrument (Waters Ltd., Elstree, UK) equipped with a nanoflow Z-spray source and calibrated with CsI $(15 \mu \mathrm{M})$. Data were analyzed using MassLynx 3.4 (Waters Ltd.) with molecular masses calculated from the centroid values of at least three charge states. The mass spectra shown represent the convolution of three charged species with minimal smoothing and converted to a mass scale.

\section{Size-exclusion chromatography to detect native interactions}

I56T lysozyme $(7 \mu \mathrm{M}, 0.1 \mathrm{M}$ sodium citrate buffer, $\mathrm{pH}$ $5.0)$ and clusterin $(8.7 \mu \mathrm{M}, 0.1 \mathrm{M}$ sodium citrate buffer, $\mathrm{pH}$ 5.0) were incubated at $37{ }^{\circ} \mathrm{C}$ for $30 \mathrm{~min}$ both alone and together. Following this procedure, a $50 \mu \mathrm{l}$ sample was injected onto a BioSep-SEC-S 4000 column (Phenomenex, Sydney, Australia) using a Shimadzu HPLC System (Shimadzu Scientific Instruments, Sydney, Australia) with autosampler (model SIL-10Ai) and diode array detector (model SPD-M10AVP). The column was run at a flow-rate of $0.5 \mathrm{ml} / \mathrm{min}$. The UV traces shown are representative of three separate runs.

\section{ELISA assay to detect native interactions}

I56T lysozyme $(100 \mu \mathrm{g} / \mathrm{ml}, 0.1 \mathrm{M}$ sodium citrate buffer, $\mathrm{pH}$ 5.0) was adsorbed on to the wells of a 96-well microtiter plate for $1 \mathrm{~h}$ at $37^{\circ} \mathrm{C}$. The wells were blocked $\left(1 \mathrm{~h}, 37^{\circ} \mathrm{C}\right)$ with HDC $(10 \mathrm{mM}$ phosphate, $150 \mathrm{mM} \mathrm{NaCl}$, $1 \%(\mathrm{w} / \mathrm{v})$ heat-denatured casein, $0.04 \%(\mathrm{w} / \mathrm{v})$ thymol, $\mathrm{pH}$ 7.4). Aliquots $(100 \mu \mathrm{l})$ of purified clusterin $(10 \mu \mathrm{g} / \mathrm{ml}$ in HDC) were added to these wells, incubated for $1 \mathrm{~h}$ at $37^{\circ} \mathrm{C}$, and then washed repeatedly with PBS to remove any unbound clusterin. Bound clusterin was detected using a mixture of purified G7 and 41D mouse antihuman-clusterin monoclonal antibodies ( $5 \mu \mathrm{g} / \mathrm{ml}$ of each), followed by treatment with an anti-mouse IgG secondary antibody conjugated to alkaline phosphatase (WesternBreeze Chromogenic Immunodetection System, Invitrogen UK). Detection was performed spectrometrically at $405 \mathrm{~nm}$, after addition of $p$-nitrophenol phosphate.

\section{ELISA pull-down assay to detect lysozyme/clusterin complex}

Purified G7 anti-clusterin antibody ( $5 \mu \mathrm{g} / \mathrm{ml}$ in PBS) or I59T human lysozyme $(0.1 \mathrm{mg} / \mathrm{ml})$ was adsorbed on to the wells of a 96-well microtiter plate $\left(1 \mathrm{~h}, 37^{\circ} \mathrm{C}\right)$, followed by washing six times with PBS. The wells were 
blocked using the Westernbreeze (Invitrogen UK) blocking reagent (casein and detergent) $\left(1 \mathrm{~h}, 37^{\circ} \mathrm{C}\right)$. Timepoints from a lysozyme aggregation reaction in the presence of a 1:8 molar ratio of clusterin to lysozyme were incubated in the wells $\left(1 \mathrm{~h}, 37^{\circ} \mathrm{C}\right)$, biotinylated clusterin was incubated as a positive control for clusterin binding, and lysozyme $(0.1 \mathrm{mg} / \mathrm{ml})$ was incubated as a positive control for lysozyme detection. The wells were washed six times with PBS followed by incubation with a 1:1000 dilution of anti-lysozyme antibody or a 1:2000 dilution of streptavidin-alkaline phosphatase (for biotinylated clusterin). The wells were again washed with PBS (six times) and incubated for $1 \mathrm{~h}$ at $37{ }^{\circ} \mathrm{C}$ with an antirabbit IgG secondary antibody conjugated to alkaline phosphatase. The wells were washed six times with Trisbuffered saline. Detection was performed with the ELISA amplification kit (Invitrogen UK), following the manufacturer's instructions.

\section{Acknowledgements}

The authors would like to thank Dr. Marcos Alcocer, Dr. Andrew Spencer and Prof. David Archer for assistance with protein expression. J.R.K. was supported in part by an NSERC (Canada) Postdoctoral Fellowship, G.L.C. was supported by an EPSRC studentship and C.L.H. was supported by a Churchill Scholarship. J.J.Y. is supported by an Australian Postgraduate Research Award and E.M.S. is supported by a University of Wollongong Matching Scholarship. M.R.W. is supported by the University of Wollongong, the Center for Medical Bioscience and an Australian Research Council Discovery Project grant (DP0773555). C.V.R. would like to thank the Walters-Kundert Trust fund. The research of C.M.D. is supported, in part, by Programme Grants from the Wellcome Trust and the Leverhulme Trust.

\section{References}

1. Dobson, C. M. (1999). Protein misfolding, evolution and disease. Trends Biochem. Sci. 24, 329-332.

2. Pepys, M. B., Hawkins, P. N., Booth, D. R., Vigushin, D. M., Tennent, G. A., Soutar, A. K. et al. (1993). Human lysozyme gene mutations cause hereditary systemic amyloidosis. Nature, 362, 553-557.

3. Yazaki, M., Farrell, S. A. \& Benson, M. D. (2003). A novel lysozyme mutation Phe57lle associated with hereditary renal amyloidosis. Kidney Int. 63, 1652-1657.

4. Valleix, S., Drunat, S., Philit, J. B., Adoue, D., Piette, J. C., Droz, D. et al. (2002). Hereditary renal amyloidosis caused by a new variant lysozyme W64R in a french family. Kidney Int. 61, 907-912.

5. Röcken, C., Becker, K., Fändrich, M., Schroeckh, V., Stix, B., Rath, T. et al. (2005). ALys amyloidosis caused by compound heterozygosity in exon 2 (Thr70Asn) and exon 4 (Trp112Arg) of the lysozyme gene. Hum. Mutat. 27, 119-120.

6. Booth, D. R., Pepys, M. B. \& Hawkins, P. N. (2000). A novel variant of human lysozyme $(\mathrm{T} 70 \mathrm{~N})$ is common in the normal population. Hum. Mutat. 16, 180.
7. Booth, D. R., Sunde, M., Bellotti, V., Robinson, C. V., Hutchinson, W. L., Fraser, P. E. et al. (1997). Instability, unfolding and aggregation of human lysozyme variants underlying amyloid fibrillogenesis. Nature, 385, 787-793.

8. Canet, D., Sunde, M., Last, A. M., Miranker, A., Spencer, A., Robinson, C. V. \& Dobson, C. M. (1999). Mechanistic studies of the folding of human lysozyme and the origin of amyloidogenic behavior in its disease-related variants. Biochemistry, 38, 6419-6427.

9. Canet, D., Last, A. M., Tito, P., Sunde, M., Spencer, A., Archer, D. B. et al. (2002). Local cooperativity in the unfolding of an amyloidogenic variant of human lysozyme. Nature Struct. Biol. 9, 308-315.

10. Dumoulin, M., Last, A. M., Desmyter, A., Decanniere, K., Canet, D., Larsson, G. et al. (2003). A camelid antibody fragment inhibits the formation of amyloid fibrils by human lysozyme. Nature, 424, 783-788.

11. Dumoulin, M., Canet, D., Last, A. M., Pardon, E., Archer, D. B., Muyldermans, S. et al. (2005). Reduced global cooperativity is a common feature underlying the amyloidogenicity of pathogenic lysozyme mutations. J. Mol. Biol. 346, 773-788.

12. Morozova-Roche, L. A., Zurdo, J., Spencer, A., Noppe, W., Receveur, V., Archer, D. B. et al. (2000). Amyloid fibril formation and seeding by wild-type human lysozyme and its disease-related mutational variants. J. Struct. Biol. 130, 339-351.

13. Esposito, G., Garcia, J., Mangione, P., Giorgetti, S., Corazza, A., Viglino, P. et al. (2003). Structural and folding dynamics properties of T70N variant of human lysozyme. J. Biol. Chem. 278, 25910-25918.

14. Johnson, R. J. K., Christodoulou, J., Dumoulin, M., Caddy, G., Alcocer, M. J., Murtagh, G. J. et al. (2005). Rationalising lysozyme amyloidosis: Insight from the structure and solution dynamics of T70N lysozyme. J. Mol. Biol. 352, 823-836.

15. Harper, J. D. \& Lansbury, P. T. (1997). Models of amyloid seeding in Alzheimer's disease and scrapie: mechanistic truths and physiological consequences of the time-dependent solubility of amyloid proteins. Annu. Rev. Biochem. 66, 385-407.

16. Dobson, C. M. (2003). Protein folding and misfolding. Nature, 426, 884-890.

17. Pepys, M. B. (2006). Amyloidosis. Annu. Rev. Med. 57, 223-241.

18. Muchowski, P. J. \& Wacker, J. L. (2005). Modulation of neurodegeneration by molecular chaperones. Nature Rev. Neurosci. 6, 11-22.

19. Yerbury, J. J., Stewart, E. M., Wyatt, A. R. \& Wilson, M. R. (2005). Quality control of protein folding in extracellular space. EMBO Rep. 6, 1-6.

20. Chiti, F. \& Dobson, C. M. (2006). Protein misfolding, functional amyloid, and human disease. Annu. Rev. Biochem. 75, 333-366.

21. Kapron, J. T., Hilliard, G. M., Lakins, J. N., Tenniswood, M. P. R., West, K. A., Carr, S. A. \& Crabb, J. W. (1997). Identification and characterization of glycosylation sites in human serum clusterin. Protein Sci. 6, 2120-2133.

22. Jones, S. E. \& Jomary, C. (2002). Clusterin. Intl J. Biochem. Cell Biol. 34, 427-431.

23. Jenne, D. E., Lowin, B., Peitsch, M. C., Böttcher, A., Schmitz, G. \& Tschopp, J. (1991). Clusterin (complement lysis inhibitor) forms a high density lipoprotein complex with apolipoportein A-I in human plasma. J. Biol. Chem. 266, 11030-11036. 
24. Murphy, B. F., Kirszbaum, L., Walker, I. D. \& d'Apice, A. J. F. (1988). SP-40,40 a newly identified normal human serum protein found in the SC5b-9 complex of complement and in the immune deposits in glomerulonephritis. J. Clin. Invest. 81, 1858-1864.

25. Shannan, B., Seifert, M., Leskov, K., Willis, J., Boothman, D., Tilgen, W. \& Reichroth, J. (2006). Challenge and promise: roles for clusterin in pathogenesis, progression and therapy of cancer. Cell Death Differ. 13, 12-19.

26. Humphreys, D. T., Carver, J. A., Easterbrook-Smith, S. B. \& Wilson, M. R. (1999). Clusterin has chaperonelike activity similar to that of small heat shock proteins. J. Biol. Chem. 274, 6875-6881.

27. Carver, J. A., Rekas, A., Thorn, D. C. \& Wilson, M. R. (2003). Small heat-shock proteins and clusterin: Intraand extracellular molecular chaperones with a common mechanism of action and function? IUBMB Life, 55, 661-668.

28. Dumoulin, M., Bellotti, V. \& Dobson, C. M. (2005). Hereditary systemic amyloidosis associated with mutational variants of human lysozyme. In Amyloid Proteins: The Beta Pleated Sheet Conformation and Disease (Sipe, J., ed), pp. 635-656, Wiley-VCH Verlag GmbH and Co. KgaA publisher, Weinheim.

29. Bader, R., Bamford, R., Zurdo, J., Luisi, B. F. \& Dobson, C. M. (2006). Probing the mechanism of amyloidogenesis through a tandem repeat of the PI3-SH3 domain suggests a generic model for protein aggregation and fibril formation. J. Mol. Biol. 356, 189-208.

30. Hatters, D. M., Lindner, R. A., Carver, J. A. \& Howlett, G. J. (2001). The molecular chaperone, alpha-crystallin, inhibits amyloid formation by apolipoprotein C-II. J. Biol. Chem. 276, 33755-33761.

31. Hatters, D. M., Wilson, M. R., Easterbrook-Smith, S. B. \& Howlett, G. J. (2002). Suppression of apolipoprotein C-II amyloid formation by the extracellular chaperone, clusterin. Eur. J. Biochem. 269, 2789-2794.
32. Dumoulin, M., Kumita, J. R. \& Dobson, C. M. (2006). Normal and aberrant biological self-assembly: insights from studies of human lysozyme and its amyloidogenic variants. Accts Chem. Res. 39, 603-610.

33. Treweek, T. M., Morris, A. M. \& Carver, J. A. (2003). Intracellular protein unfolding and aggregation: the role of small heat-shock chaperone proteins. Aust. J. Chem. 56, 357-367.

34. Ohashi, K. (2001). Pathogenesis of $\beta_{2}$-microglobulin amyloidosis. Pathol. Int. 51, 1-10.

35. Cohen, E., Bieschke, J., Perciavalle, R. M., Kelly, J. W. \& Dillin, A. (2006). Opposing activities protect against age-onset proteotoxicity. Science, 313, 1604-1610.

36. Spencer, A., Morozova-Roche, L. A., Noppe, W., MacKenzie, D. A., Jeenes, D. J., Joniau, M. et al. (1999). Expression, purification, and characterization of the recombinant calcium-binding equine lysozyme secreted by the filamentous fungus Aspergillus niger: comparisons with the production of hen and human lyosyzmes. Protein Expr. Purif. 16, 171-180.

37. Kumita, J. R., Johnson, R. J. K., Alcocer, M. J. C., Dumoulin, M., Holmqvist, F., McCammon, M. G. et al. (2006). Impact of the native-state stability of human lysozyme variants on protein secretion by Pichia pastoris. FEBS J. 273, 711-720.

38. Wilson, M. R. \& Easterbrook-Smith, S. B. (1992). Clusterin binds by a multivalent mechanism to the Fc and Fab regions of IgG. Biochim. Biophys. Acta, 1159, 319-326.

39. Lakins, J. N., Poon, S., Easterbrook-Smith, S. B., Carver, J. A., Tenniswood, M. P. \& Wilson, M. R. (2002). Evidence that clusterin has discrete chaperone and ligand binding sites. Biochemistry, 41, 282-291.

40. Yerbury, J. J., Poon, S., Meehan, S., Thompson, B., Kumita, J. R., Dobson, C. M. et al. (2007). Clusterin influences amyloid formation and toxicity by interacting with pre-fibrillar structures. FASEB J.; In the press.

Edited by S. Radford 